Supporting Information to

\title{
An Easy Way to Sugar-containing Polymer Vesicles or Glycosomes
}

\author{
Liangchen You and Helmut Schlaad* \\ Max Planck Institute of Colloids and Interfaces \\ Research Campus Golm, 14424 Potsdam, Germany
}

Materials. 1,3-Butadiene (Sigma-Aldrich) was first distilled from dibutylmagnesium and then from $n$-butyllithium prior to use. Styrene (Sigma-Aldrich) was distilled from $\mathrm{CaH}_{2}$ and from dibutylmagnesium. Tetrahydrofuran (THF) (BASF AG, Ludwigshafen) was fractionated and distilled twice from $\mathrm{Na} / \mathrm{K}$ alloy. sec-Butyllithium (sBuLi) (1 M solution in hexane, Sigma-Aldrich) was used as received.

2,3,4,6-Tetra-O-acetyl- $\beta$-D-1-thioglucopyranose (2) (97\%, Sigma-Aldrich; see chemical structure and ${ }^{1} \mathrm{H}$ NMR spectrum in Figure SI-1) and azoisobutyronitrile (AIBN) (98\%, Fluka) were used as received. Chloroform (99\%, Roth) and THF (99.5\%, Fluka) were distilled from $\mathrm{CaH}_{2}$, and methanol (99.9\%, Merck) was distilled from magnesium turnings. $0.5 \mathrm{M}$ solution of sodium methoxide in methanol was prepared by adding $0.115 \mathrm{~g}$ sodium (lump in kerosene, $99 \%$, Sigma-Aldrich) into $10 \mathrm{~mL}$ of dry methanol. Amberlite $200 \mathrm{C}$ ion exchange resin (Fluka) was washed with $0.5 \mathrm{~N} \mathrm{NaOH}$, water, $0.5 \mathrm{~N} \mathrm{HCl}$, and water before use.

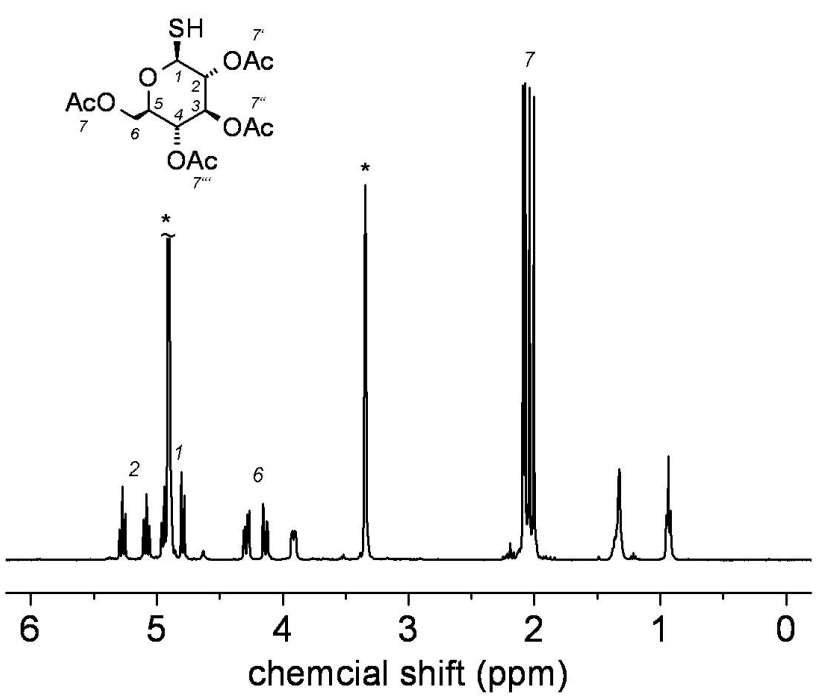

Figure SI-1. Chemical structure and ${ }^{1} \mathrm{H}$ NMR spectrum $\left(400.1 \mathrm{MHz}, \mathrm{CD}_{3} \mathrm{OD}\right)$ of 2.

Anionic polymerization. 1,3-Butadiene was condensed into a reactor containing a mixture of THF and sBuLi, and the solution was stirred overnight at $-78{ }^{\circ} \mathrm{C}$. After withdrawal of an aliquot from the reactor, styrene was condensed into the reactor and polymerized for 1 hour at the same temperature. The reaction was quenched with degassed methanol. The 1,2-polybutadiene-block-polystyrene (1) was precipitated into methanol, filtered, and dried under vacuum at $+40^{\circ} \mathrm{C}$. 
Photoaddition. A solution of $100 \mathrm{mg}$ of $\mathbf{1}, 450 \mathrm{mg}$ of $\mathbf{2}$, and $11 \mathrm{mg}$ of AIBN in $6 \mathrm{~mL}$ of dry THF was degassed by three freezepump-thaw cycles and then put under an argon atmosphere. After stirring for $5 \mathrm{~h}$ under irradiation with a mercury lamp, most solvent was removed and the crude product was isolated by precipitation into $\mathrm{MeOH}$ and filtration. Product $3 \mathbf{a}$ was purified by dissolving in THF and precipitating into $\mathrm{MeOH}$ twice.
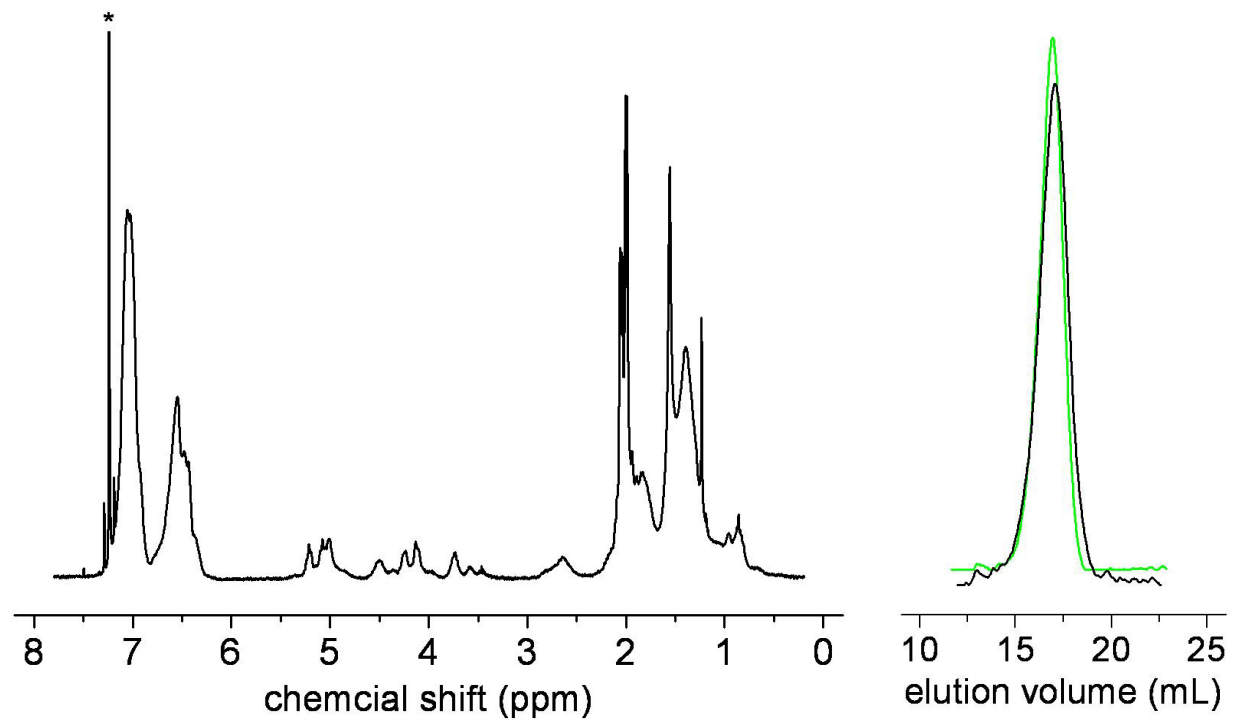

Figure SI-2. Left: ${ }^{1} \mathrm{H}$ NMR spectrum $\left(400.1 \mathrm{MHz}, \mathrm{CDCl}_{3}\right)$ of 3a. Right: SEC chromatograms $\left(\mathrm{CHCl}_{3}\right.$, detector: $\left.\mathrm{RI}\right)$ of $\mathbf{3 a}$ (black) and the precursor polymer 1 (green).

Deacetylation. A solution of $100 \mathrm{mg}$ of $\mathbf{3 a}$ in $9 \mathrm{~mL}$ chloroform and $1 \mathrm{~mL}$ of $0.5 \mathrm{M}$ sodium methoxide in methanol was stirred for $1 \mathrm{~h}$ at room temperature. After neutralization with Amberlite $200 \mathrm{C}$ ion exchange resin, filtration, and evaporation of solvent, product $\mathbf{3 b}$ was suspended in water and freeze-dried.

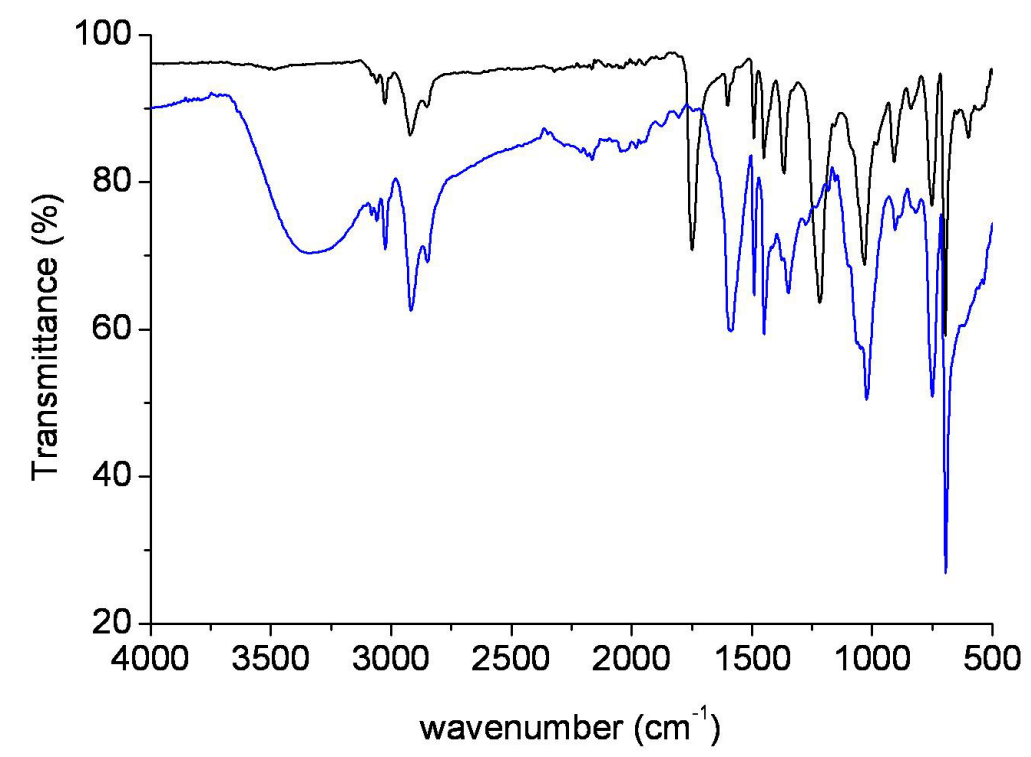

Figure SI-3. FT-IR spectra of $\mathbf{3 a}$ (black) and $\mathbf{3 b}$ (blue) (solid samples). 
Sample preparation for structural analysis. $1 \mathrm{~mL}$ of a $0.3 \mathrm{wt} \%$ solution of the glucose-grafted 1,2-polybutadiene-blockpolystyrene (3b) in THF ( $+20 \mu \mathrm{L}$ of water) was stirred at room temperature for $24 \mathrm{~h}$. Deionized water was added slowly to the solution until the water content reached $50 \mathrm{wt} \%$. The solution was then stirred in the open vial to remove the THF until constant weight. This stock solution was then diluted with water by a factor of 100. For Dynamic Light Scattering (DLS) measurements, the sample was passed through a $5 \mu \mathrm{m}$ filter (Schleicher-Schüll). For visualization of aggregates with Transmission Electron Microscopy (TEM), a drop of the solution was deposited onto a copper grid coated with a thin film of Formvar and a film of carbon. After 10 minutes, the excess solution was absorbed by a piece of filter paper, and the grid was dried in the air overnight.

Analytical methods and instrumentation. C/H/N/S-specific Elemental Analysis was done with a Vario EL Elemental Analyzer. ${ }^{1} \mathrm{H}$ NMR spectra were recorded at room temperature with a Bruker DPX-400 spectrometer operating at $400.1 \mathrm{MHz}$. Fouriertransform infrared (FT-IR) spectroscopy was done at room temperature with a BioRad 6000 FT-IR spectrometer equipped with a Single Reflection Diamond ATR. Size exclusion chromatography (SEC) with simultaneous UV and RI detection was performed in chloroform at $25^{\circ} \mathrm{C}$ using a column set of two $300 \times 8 \mathrm{~mm}$ MZ-SDplus (spherical polystyrene particles with an average diameter of $5 \mu \mathrm{m}$ ) columns with pore sizes of $10^{3}$ and $10^{6} \AA$. Calibration was done with commercial polystyrene standards. Dynamic Light Scattering (DLS) measurements were carried out on a home-built goniometer with a fixed scattering angle of $90^{\circ}$ at $25^{\circ} \mathrm{C}$. The instrument was connected via a single mode fiber to a single photon detector (ALV/SOSIPD), the output of which was fed to a multiple-tau digital correlator (ALV 5000). The light source (Polytec, $34 \mathrm{~mW}$ ) was a $\mathrm{HeNe}$ laser operating at a wavelength of $\lambda=633 \mathrm{~nm}$. From the measured time-correlation functions, intensity-weighted particle size distributions were calculated according to $\mathrm{H}$. Schnablegger and O. Glatter, Appl. Opt. 1991, 30, 4889-4896. Transmission Electron Microscopy (TEM) was performed on Zeiss EM 912 Omega microscope at an acceleration voltage of $120 \mathrm{kV}$. 

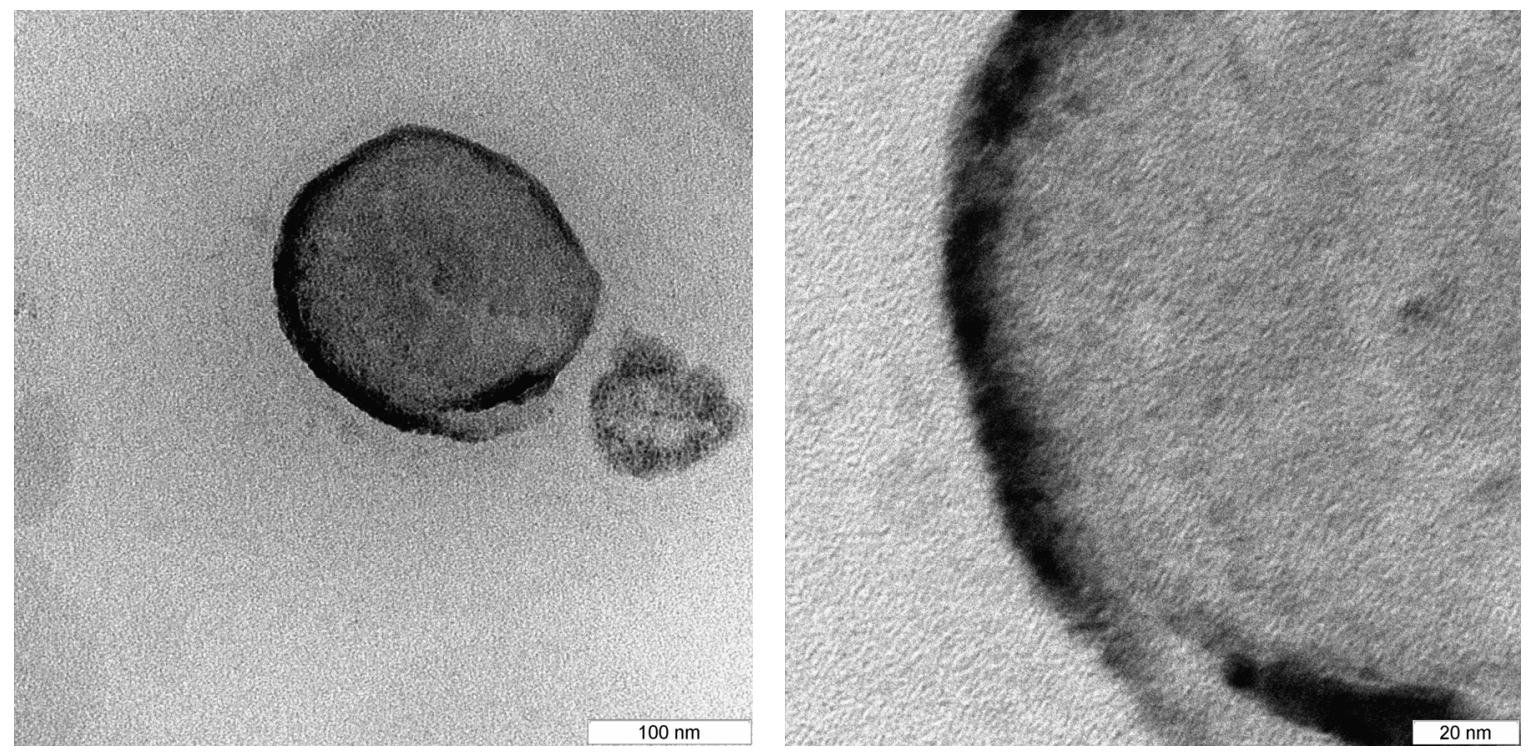

Figure SI-4. TEM images of a collapsed vesicle of $\mathbf{3 b}$ in THF. Specimen was prepared by placing a drop of a 0.1 wt $\%$ polymer solution on a deep-frozen grid and allowing the solvent to evaporate in air.
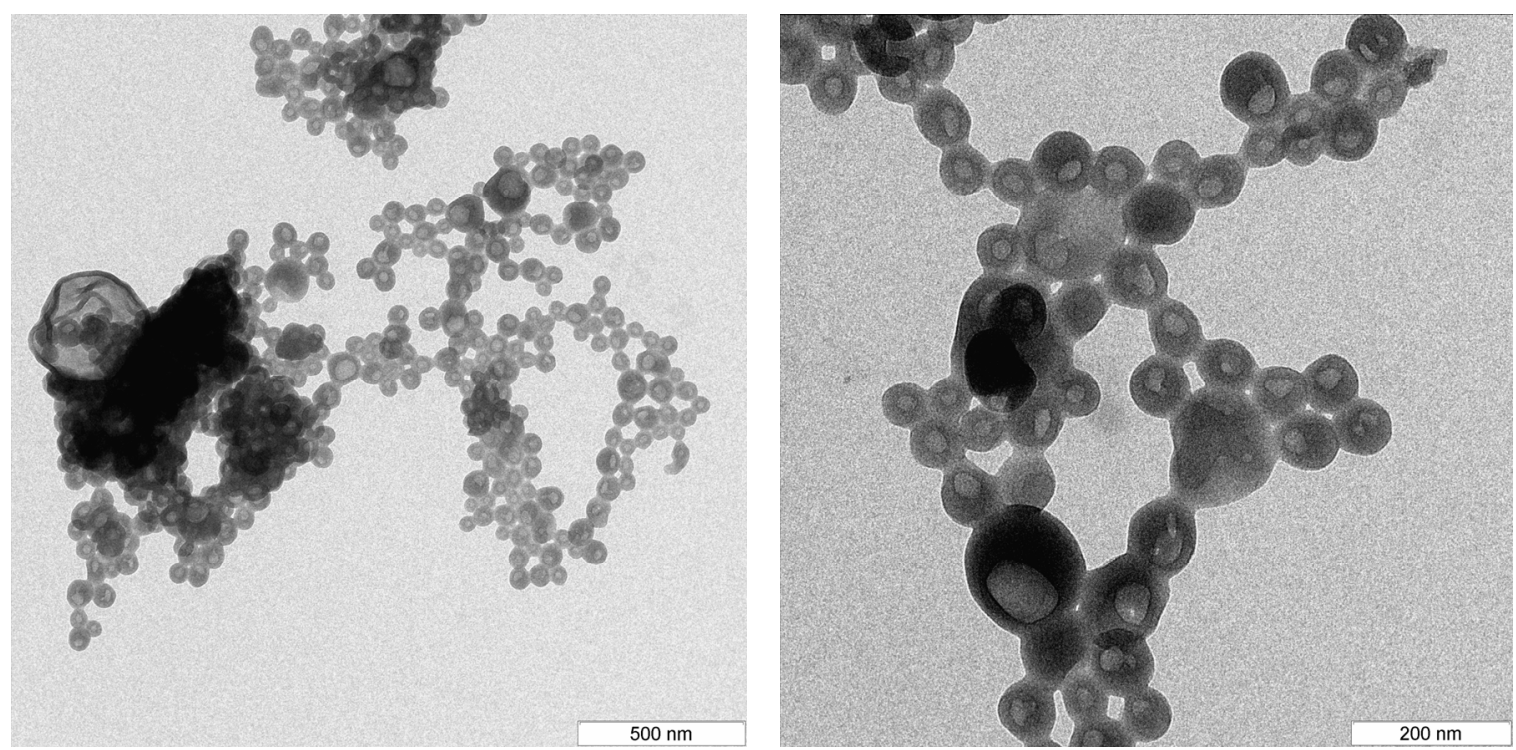

Figure SI-5. TEM images of (collapsed) vesicles of $\mathbf{3 b}$ in water. Specimen was prepared by placing a drop of a 0.003 wt $\%$ polymer solution on a grid and allowing the solvent to evaporate in air. 
hydrophobic hydrophobic

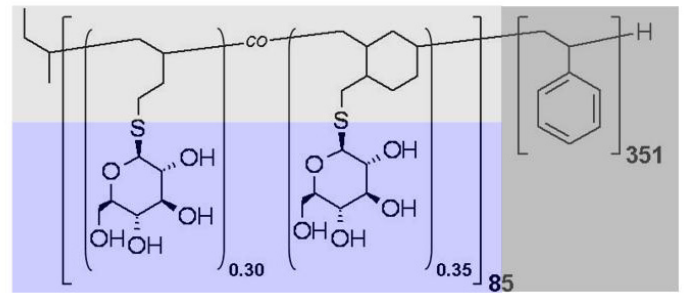

hydrophilic

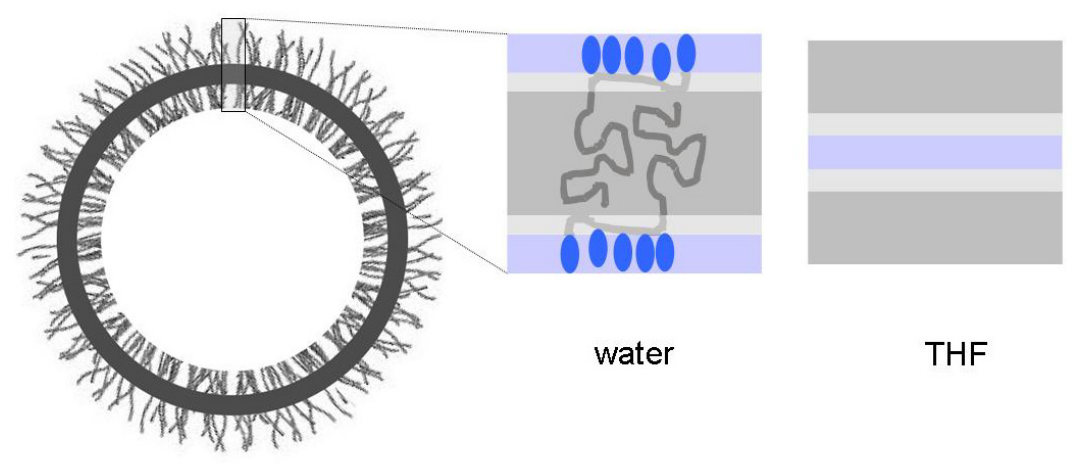

Figure SI-6. Schematic illustration of the structure of the vesicle membrane (working hypothesis). 